\title{
A comparison of numerical and analytical methods for the reduced wave equation with multiple spatial scales
}

\author{
John P. Boyd \\ Department of Atmospheric, Oceanic \& Space Physics and Laboratory for Scientific Computation, \\ University of Michigan, 2455 Hayward Avenue, Ann Arbor, MI 48109, USA
}

\section{Abstract}

Boyd, J.P., A comparison of numerical and analytical methods for the reduced wave equation with multiple spatial scales, Applied Numerical Mathematics 7 (1991) 453-479.

We compare four different techniques for solving the ordinary differential equation $u_{x x} \pm u=f(\varepsilon x)$ on the unbounded interval, $x \in[-\infty, \infty]$, when $f(\varepsilon x)$ decays rapidly as $|x|, \infty$. This problem, although very simple, is representative of problems that arise in such diverse fields as numerical weather prediction, plasma physics, and weakly non-local solitary waves. When $\varepsilon \ll 1$, the solution has two length scales: the "fast", $O(1)$ scale of the homogeneous solutions of the differential equation and the "slow", $O(1 / \varepsilon)$ scale of the forcing function. The four methods are: (1) perturbation series in $\varepsilon$ ("method of multiple scales"); (2) Padé approximants formed from the $\varepsilon$-series; (3) rational Chebyshev pseudospectral algorithm; and (4) the pseudospectral method with a mixed basis that includes a special "radiation function" for the plus sign only.

We find that the perturbation series is asymptotic but almost always divergent. The effectiveness of the other methods depends on the sign of the coefficient in the differential equation. When the sign is negative, $u(x)$ decays rapidly as $|x| \rightarrow \infty$. Padé approximants converge and the rational Chebyshev pseudospectral method is very accurate. One might suppose that the numerical method would be ineffective for small $\varepsilon$ because of the difficulty of simultaneously resolving two very disparate length scales. However, because that part of $u(x)$ which varies on the "fast" $O(1)$ scale is exponentially small in $1 / \varepsilon$, as few as twenty basis functions give six decimal place accuracy for a smooth $f(x)$ for all $\varepsilon$.

When the sign of the differential equation is negative, $u(x)$ is oscillatory as $|x| \rightarrow \infty$ (with an amplitude $\alpha$ which is proportional to $\exp (-q / \varepsilon)$ for some constant $q$ ). Padé approximants and the Chebyshev method do not converge, but instead have an accuracy which is limited to $O(\alpha)$. When a special "radiation function" is added to the spectral basis, however, it is possible to obtain arbitrarily high accuracy. The numerically computed coefficient of the radiation function is an accurate approximation to the amplitude of the asymptotic radiation, $\alpha(\varepsilon)$.

\section{Introduction}

The review by Boyd [11] shows that the key to understanding "weakly non-local" solitary waves is the simple boundary value problem

$$
u_{x x} \pm u=f(\varepsilon x), \quad x \in[-\infty, \infty] \text {. }
$$


where $u(x)$ is as small as possible as $|x| \rightarrow \infty$ and where $f(x) \rightarrow 0$ exponentially fast as $|x| \rightarrow \infty$. Table 1 lists eight examples of "non-local" phenomena drawn from such diverse fields as particle physics, numerical weather prediction, oceanography, and plasma physics. Note that (1.1) also arises in linear problems, too, such as quantum scattering. In Section 9, we explain why the special Chebyshev method introduced here is so useful in attacking these problems.

Equation (1.1) is also the prototype for the reduced linear wave equation. That is, equation (1.1) is representative of the boundary value problems that arise when a wave equation is given a forcing which is harmonic in time. The reduced wave equation is obtained by dividing the $\cos (\omega t)$ time dependence from both forcing and unknown and then rescaling the coordinates so that $\omega=1$. (The connection between (1.1) and the large-time solution of the time-dependent wave equation is discussed further in Appendix A.)

Lastly, (1.1) is interesting because the $\varepsilon$-series for $u(x ; \varepsilon)$ is-thanks to the absence of boundaries (and boundary layers) at finite $|x|$-the simplest nontrivial illustration of the "method of multiple scales", which has been one of the cornerstones of applied mathematics $[17,23]$. In Sections 3-5, we do not merely prove that the perturbation series is usually divergent, as in the standard textbooks, but also explain why, and also why sometimes even Padé approximants fail.

The asymptotic behavior of $u(x)$ is quite different depending on the sign of the undifferentiated term in the differential equation. When the sign is negative, which we shall henceforth call "Case 1", $u(x)$ decays rapidly for large $|x|$, exponentially fast if $f(x)$ decays exponentially. This means that one may apply the unambiguous boundary condition $u(x) \rightarrow 0$ as $|x| \rightarrow \infty$ and a special pseudospectral basis is unnecessary. Nevertheless, we shall see in Section 3 that even for

Table 1

Examples of slowly-varying oscillations with exponentially small "far field" radiation

\begin{tabular}{|c|c|c|}
\hline Name & Field & Reference \\
\hline $\begin{array}{l}\text { Water waves with surface tension } \\
\text { (generalized Korteweg-de Vries) }\end{array}$ & Hydrodynamics & $\begin{array}{l}\text { Pomeau et al. [25] } \\
\text { Hunter-Scheurle [16] } \\
\text { Boyd [12] }\end{array}$ \\
\hline Higher-mode Rossby solitons & $\begin{array}{l}\text { Meteorology } \\
\text { Oceanography }\end{array}$ & Boyd $[8,11]$ \\
\hline Plasma modons in magnetic shear & Plasma Physics & Meiss-Horton [20] \\
\hline "Slow manifold" & Meteorology & $\begin{array}{l}\text { Lorenz-Krishnamurthy }[18] \\
\text { Boyd }[7,11]\end{array}$ \\
\hline$\phi^{4}$ breather & Particle Physics & $\begin{array}{l}\text { Segur-Kruskal }[26] \\
\text { Boyd }[7,10,11]\end{array}$ \\
\hline Island-trapped waves & Oceanography & $\begin{array}{l}\text { Lozano-Meyer [19], } \\
\text { Meyer [21] }\end{array}$ \\
\hline Quantum scattering & Physics & $\begin{array}{l}\text { Pokrovskii-Khalatnikov [24] } \\
\text { Boyd [9] }\end{array}$ \\
\hline $\begin{array}{l}\text { Dendrite formation/ } \\
\text { Taylor-Saffman problem }\end{array}$ & Fluid Mechanics & $\begin{array}{l}\text { Hong-Langer [15] } \\
\text { Combescot et al. [13] }\end{array}$ \\
\hline
\end{tabular}


this straightforward case, the perturbation series is divergent and all numerical methods must confront disparate length scales.

When the sign in (1.1) is positive-henceforth called "Case 2"- $-u(x)$ does not asymptote to a constant but rather to an oscillation. The two cases are contrasted in Fig. 1. The strong boundary condition $u( \pm \infty)=0$ must be replaced by the weaker condition that $u(x)$ is bounded at infinity. If $u(x)$ is a solution to (1.1), then

$$
v(x) \equiv u(x)+\gamma \cos (x)+\delta \sin (x)
$$

is also a bounded solution for arbitrary constants $\gamma$ and $\delta$. The physics of the problem determines $\gamma$ and $\delta$. In Section 7, we shall see that it is possible to build $\gamma$ and $\delta$ into the numerical algorithm to solve (1.1) for the physically relevant solution.

Nevertheless, the plus sign in (1.1) is more complicated than the case $u_{x x}-u=f(\varepsilon x)$. Not only the perturbation series but also the corresponding Padé approximants are non-convergent. Furthermore, all standard numerical methods are defeated by the asymptotic oscillation. In Section 7, we solve (1.1) by making a novel and unconventional modification to the spectral basis set.

One consolation is that the amplitude of the "far field" oscillation is exponentially small in $1 / \varepsilon$. This would suggest that one might, at least for sufficiently small $\varepsilon$, ignore the oscillation completely. Unfortunately, this amplitude in its very smallness is often crucial in applications. Segur and Kruskal [26] have shown that the "far field" oscillation is the reason that the $\phi^{4}$ breather is not a true soliton. It decays with time via radiation to infinity; by computing the far field oscillation, one can estimate the radiative lifetime. Similarly, Lorenz and Krishnamurthy [18] have shown that, at least in their simple model, a similar oscillation wrecks the hypothesized "slow manifold" which is fundamental to initializing weather forecasting models. Thus, calculating the far field oscillation is and must be an important priority.

In the next section, we briefly review the analytical solution to (1.1) for comparison with the four general algorithms discussed in the rest of the paper. Section 3 describes the most crucial feature of the analytical solutions: the rapid variations of $u(x)$ on the "fast", $\mathrm{O}(1)$ length scale, are exponentially small in $1 / \varepsilon$. Section 4 derives the perturbation series and explains why it

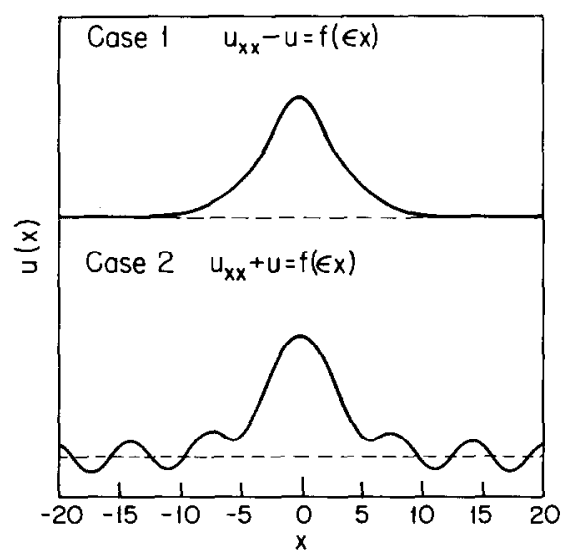

Fig. 1. Schematic showing the differences between Case 1 and Case 2. The radiation coefficient $\alpha$ is the amplitude of the oscillations for large $|x|$ in the lower diagram. 
diverges. The next part of the article is a numerical study of Padé approximants. Although it is very difficult to prove rigorous Padé theorems, the numerical evidence strongly supports the conclusion given above: Padé approximants are useful, but only for the negative sign in (1.1). Sections 6 and 7 describe the Chebyshev numerical method and the successful trick which is needed to handle the plus sign with its asymptotic oscillation. In Section 8, we generalize the constant coefficient boundary value problem to variable coefficients. The answer is that neither the qualitative analysis nor the numerical algorithms are altered provided that the differential equation coefficients vary on the same slow $\mathrm{O}(1 / \varepsilon)$ length scale as the forcing function. Section 9 shows that the radiation coefficient, i.e., the strength of the far field oscillation, is very sensitive to perturbations in the forcing, $f(\varepsilon x)$. This is why the Chebyshev/radiation basis algorithm is so important in solving non-local wave equations. The final section is a catalogue of extensions and open problems. Lastly, Appendix A shows how the solutions of the time-dependent wave equation asymptote to the solution of the reduced wave equation, (1.1).

\section{Analytical solutions}

Taking the Fourier transform of both sides of (1.1) gives, after elementary algebra, the solution to $u_{x x} \pm u=f(\varepsilon x)$ as

$$
u(x ; \varepsilon)=\left[ \pm 1 / \varepsilon(2 \pi)^{1 / 2}\right] \int_{-\infty}^{\infty} \mathrm{d} k F(k / \varepsilon) \mathrm{e}^{\mathrm{i} k x} /\left(1 \mp k^{2}\right)
$$

where $F(k / \varepsilon)$ is the Fourier transform of the forcing function:

$$
F(k) \equiv\left[1 /(2 \pi)^{1 / 2}\right] \int_{-\infty}^{\infty} \mathrm{d} x f(x) \mathrm{e}^{-\mathrm{i} k x}
$$

For Case 1, which is the lower sign in (2.1), the Fourier representation is nonsingular and completely satisfactory. When the upper sign in (2.1) applies, however, the integrand is infinite at $k= \pm 1$. The representation is still valid, but the integral cannot be evaluated without specifying how the contour is deformed above or below or through these poles at $k= \pm 1$. The ambiguity represented by this choice of contour reflects the ambiguity inherent in the phase constants $\gamma$ and $\delta$ in (1.2): one may add arbitrary multiples of $\cos (x)$ and $\sin (x)$ to the integral in (2.1) (for a given choice of integration contour) and still obtain a bounded solution to (1.1).

For Case 2 (asymptotic oscillation), it is therefore convenient to use the alternative integral representation

$$
\begin{aligned}
u(x ; \varepsilon)= & \frac{1}{2} \sin (x)\left\{\int_{-\infty}^{x} f(\varepsilon k) \cos (k) \mathrm{d} k-\int_{x}^{\infty} f(\varepsilon k) \cos (k) \mathrm{d} k\right\} \\
& -\frac{1}{2} \cos (x)\left\{\int_{-\infty}^{x} f(\varepsilon k) \sin (k) \mathrm{d} k-\int_{x}^{\infty} f(\varepsilon k) \sin (k) \mathrm{d} k\right\}
\end{aligned}
$$

which may be derived via variation of parameters.

In the limit that $|x| \gg 1$, which we shall henceforth call the "far field", (2.3) simplifies to

$$
u(x ; \varepsilon)=\operatorname{sgn}(x)[\alpha(\varepsilon) \sin (x)-\beta(\varepsilon) \cos (x)], \quad x \rightarrow+\infty,
$$


where

$$
\operatorname{sgn}(x) \equiv \begin{cases}1, & x \geqslant 0 \\ -1, & x<0\end{cases}
$$

and the "radiation coefficients" $\alpha(\varepsilon)$ and $\beta(\varepsilon)$ are defined by

$$
\begin{aligned}
& \alpha(\varepsilon) \equiv \int_{0}^{\infty} f_{\mathrm{S}}(\varepsilon k) \cos (k) \mathrm{d} k, \\
& \beta(\varepsilon) \equiv \int_{0}^{\infty} f_{\mathrm{A}}(\varepsilon k) \sin (k) \mathrm{d} k,
\end{aligned}
$$

where $f_{\mathrm{S}}(k)$ and $f_{\mathrm{A}}(k)$ are the symmetric and antisymmetric parts of the forcing function, i.e.,

$$
\begin{aligned}
f_{\mathrm{S}}(k) & \equiv \frac{1}{2}[f(k)+f(-k)], \\
f_{\mathrm{A}}(k) & \equiv \frac{1}{2}[f(k)-f(-k)] .
\end{aligned}
$$

The signum function, $\operatorname{sgn}(x)$, in $(2.4)$ is critically important because it implies that $u(x ; \varepsilon)$ as defined by (2.3) is a particular integral which does not contain any contributions from the homogeneous solutions to (1.1). The reason is that $\sin (x)$ and $\cos (x)$ solve (1.1), but $\operatorname{sgn}(x) \sin (x)$ and $\operatorname{sgn}(x) \cos (x)$ do not solve (1.1). Thus, (2.3) is a "minimum radiation" solution in the sense that adding multiples of the homogeneous solutions of (1.1) to (2.3) would increase the amplitude of the far field oscillations as either $x \rightarrow \infty$ or $x \rightarrow-\infty$.

If the forcing $f(x)$ is symmetric about the origin (i.e., $f(x)=f(-x)$ so that $\left.f_{\mathrm{A}}(x) \equiv 0\right)$, then the "minimum radiation" solution defined by (2.3) and the exponentially decaying solution for Case 1 (lower signs in (2.1)), are also symmetric about the origin. For simplicity, we shall take $f(x)$ to be symmetric in the rest of this article so that $\beta=0$ and we need to compute only a single radiation coefficient, $\alpha$. However, all of the methods discussed below are equally applicable to solutions that are antisymmetric with respect to $x=0$ or have no definite parity.

\section{The exponential smallness of variations on the fast length scale}

One major numerical challenge of (1.1) is that the solution varies on two length scales: the $\mathrm{O}(1 / \varepsilon)$ scale of $f(\varepsilon x)$ and the $\mathrm{O}(1)$ scale of the homogeneous solutions of (1.1). For both Case 1 and Case 2, one might suppose that one would be forced to use a huge number of degrees of freedom to resolve both length scales in the limit $\varepsilon \rightarrow 0$. Clearly, the grid spacing must be $O(1)$ to resolve the "fast", $\mathrm{O}(1)$ scale. However, the computational grid must be very wide when $\varepsilon \ll 1$ because $f(\varepsilon x)$ is very wide. The number of degrees of freedom would seem to be $O(10 / \varepsilon)$ to achieve even moderate accuracy.

In this section, we show that this expectation is false because the $O(1)$ variability is exponentially small in $1 / \varepsilon$ in comparison to the slowly varying portions of $u(x ; \varepsilon)$.

Lemma 3.1. Let $f(x)$ be analytic in the complex $x$-plane everywhere inside the strip

$$
-\mu<\operatorname{Im}(x)<\mu \text {. }
$$


Then the Fourier transform of $f(x), F(k)$, satisfies the following inequality for all real $k$ :

$$
|F(k)| \leqslant B \mathrm{e}^{-\mu|\operatorname{Re}(k)|}, \quad|\operatorname{Re}(k)| \rightarrow \infty .
$$

If $f(x)$ decays exponentially with $|x|$, then (3.2) can be extended from the real $k$-axis to a strip of finite width in the complex $k$-plane.

Proof. Given in [5].

For $f(\varepsilon x)=\operatorname{sech}(\varepsilon x)$, for example, the strip of analyticity is bounded by simple poles at $x=+\mathrm{i} \pi /(2 \varepsilon)$. Substituting $\mu=\pi /(2 \varepsilon)$ into Lemma 3.1 implies the Fourier transform of $\operatorname{sech}(\varepsilon x)$ should decay as fast as $\exp (-[\pi / 2 \varepsilon])$ as $1 / \varepsilon \rightarrow \infty$. This in turn implies through (2.6) that the radiation coefficient for this forcing function should be bounded by a constant times $\exp (-[\pi / 2 \varepsilon])$. Table 2 confirms this.

Theorem 3.2. If $f(x)$ is analytic in a strip of width $\mu$ in the complex $x$-plane, then the radiation coefficients $\alpha(\varepsilon)$ and $\beta(\varepsilon)$ satisfy the bound

$$
\alpha(\varepsilon), \beta(\varepsilon) \leqslant B \mathrm{e}^{-\mu / \varepsilon}
$$

for some constant $B$.

Proof. Trivial application of Lemma 3.1 to (2.6) and (2.7).

If $f(x)$ is an entire function, that is, one with no singularities for any finite $x$, then the bound can be greatly tightened. A bandlimited function is a special class of entire functions defined by the following.

Definition 3.3. A function $f_{W}(x)$ is bandlimited with bandwidth $W$ if it can be represented as the truncated Fourier integral

$$
f_{W}(x)=\left(1 /[2 \pi]^{1 / 2}\right) \int_{-W}^{W} F(k) \mathrm{e}^{\mathrm{i} k x} \mathrm{~d} k
$$

Theorem 3.4. If $f_{W}(x)$ is a bandlimited function of bandwidth $W$, then

$$
\alpha(\varepsilon)=\beta(\varepsilon)=0, \quad \text { if } \varepsilon<1 / W \text {. }
$$

Proof. Insertion of Definition 3.3 into (2.6) and (2.7). Note that $\alpha=2 \operatorname{Re}[F(1 / \varepsilon)]$.

For bandlimited functions, Case 2 can be solved by the same rational Chebyshev method as for Case 1, and no special tricks are needed. Unfortunately, bandlimited functions are very special and one will rarely be this lucky in practice. However, bandlimited functions do have a useful role to play in the proof of Theorem 3.7 below.

The radiation coefficient is not the whole of the fast variability even for Case 2. Unfortunately, "variability on the fast length scale" does not have a unique definition. The reason is that the Fourier transform of $u(x ; \varepsilon)$ is nonzero for almost all wavenumbers $k$ so that the solution is, strictly speaking, varying on all possible length scales in $x$. The following definition, however, accords with common sense and is sufficient for our purposes. 


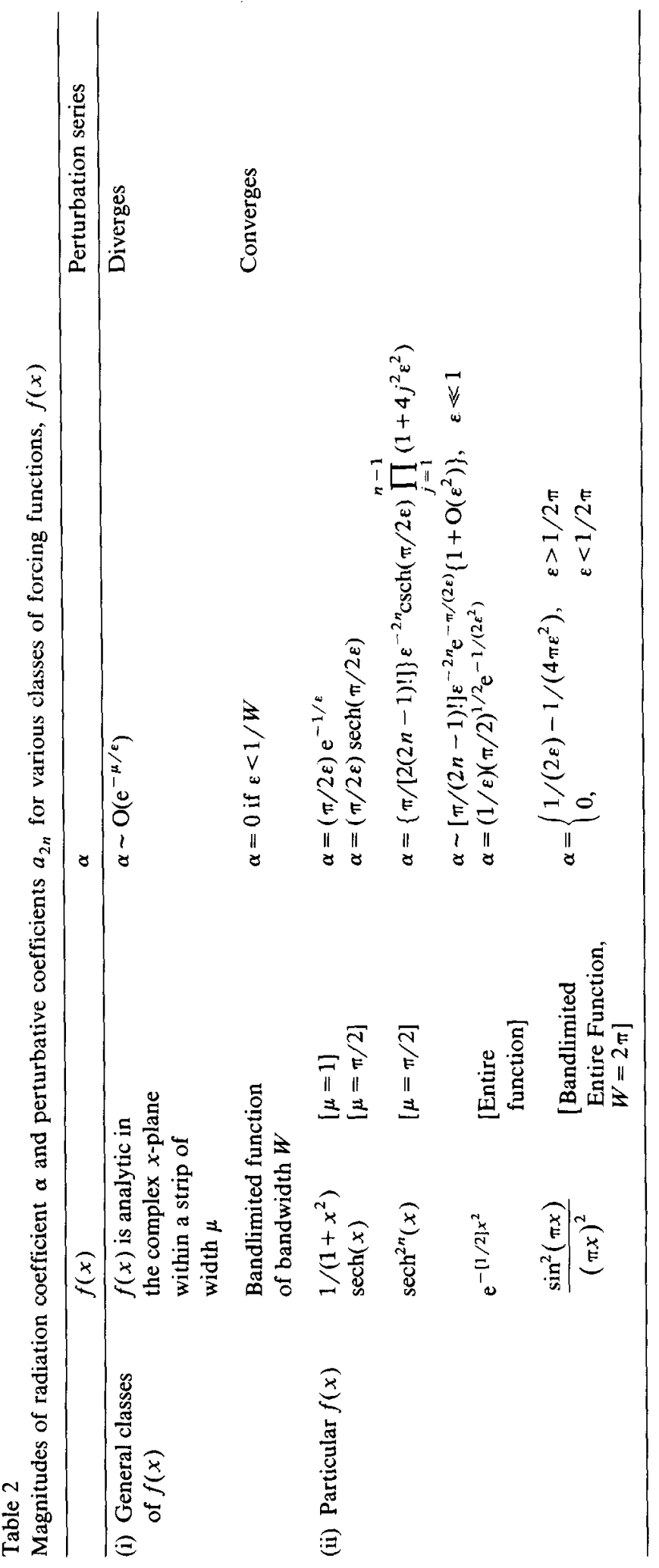


Definition 3.5. The fast scale variability is defined to be

$$
u_{\mathrm{F}}(x ; \varepsilon) \equiv \int_{-\infty}^{-1} \mathrm{~d} k U(k ; \varepsilon) \mathrm{e}^{\mathrm{i} k x}+\int_{1}^{\infty} \mathrm{d} k U(k ; \varepsilon) \mathrm{e}^{\mathrm{i} k x},
$$

where $U(k, \varepsilon)$ is the Fourier transform of $u(x ; \varepsilon)$, the solution of $u_{x x} \pm u=f(\varepsilon x)$.

Theorem 3.6. The variability on the fast, $O(1)$ length scale, $u_{\mathrm{F}}$ as defined by (3.6), satisfies the bound for both signs in (1.1)

$$
\left|u_{\mathrm{F}}(x ; \varepsilon)\right| \leqslant D \mathrm{e}^{-\mu / \varepsilon}
$$

for some constant $D$ and sufficiently small $\varepsilon$ where $f(x)$, the forcing function in (1.1), is analytic in the complex $x$-plane over a strip at least as wide as $|\operatorname{Im}(x)| \leqslant \mu$.

Proof. Lemma 3.1 provides a bound on $U(k ; \varepsilon)$, the Fourier transform of $u(x ; \varepsilon)$. Inserting this bound into the definition of $u_{\mathrm{F}}$ and the integral representations for $u(x ; \varepsilon),(2.1)$ and (2.3), and integrating gives (3.7). Strictly speaking, one should replace $\mu$ in (3.3) and (3.7) by $\mu^{\prime}=\mu-\delta$ where $\delta$ is an arbitrarily small parameter so as to compensate for miscellaneous powers of $\varepsilon$ multiplying the exponential. For simplicity, we have ignored this subtlety in stating the theorem.

There is nothing sacred about the limiting the integrals in (3.6) to $|k| \geqslant 1$; one could extend the integrals to $k= \pm 1 / 2$ at the price of weakening the bound in (3.7) to $\exp (-\mu / 2 \varepsilon)$. Regardless of precisely how one defines the variability, however, it is exponentially small in $1 / \varepsilon$.

The other issue is whether the fast scale variations in $u(x ; \varepsilon)$, small though they be, corrupt the numerical solution of (1.1). For Case 1 (no far field oscillations) and the particular numerical algorithm known as the sinc pseudospectral method, it is possible to give a particularly simple answer.

Theorem 3.7. To approximate $u(x ; \varepsilon)$, the solution to $u_{x x}-u=f(\varepsilon x)$, to within an error no worse than $\exp (-\Lambda)$ using the sinc pseudospectral method, that is,

$$
\max _{x \in[-\infty, \infty]}\left|u(x ; \varepsilon)-u_{\text {sinc }}(x ; \varepsilon)\right|<\mathrm{e}^{-\Lambda},
$$

where $\Lambda$ is an arbitrary specified error tolerance, it is sufficient to use a uniform grid spacing

$$
h<\pi \mu /(\Lambda \varepsilon),
$$

where $\mu$ is the width of the strip of analyticity of $f(x)$ in the complex $x$-plane.

Proof. This follows from three lemmas below which are all proved in Stenger [27].

Lemma 3.8. If a function $f(x)$ is analytic within the strip $|\operatorname{Im}(x)|<\mu$, then it may be approximated by a bandlimited function $f_{W}(x)$ of bandwidth $W$ with an error which decreases exponentially fast with $W$, i.e.,

$$
\left|f(x)-f_{W}(x)\right| \leqslant D \mathrm{e}^{-\mu W}
$$

for some constant $D$ and sufficiently large $W$. 
Lemma 3.9 (Shannon-Whittaker Sampling Theorem). The approximation of a bandlimited function by the sinc series

$$
u_{W}(x) \approx u_{\mathrm{sinc}}(x) \equiv \sum_{j=-\infty}^{\infty} u_{W}(j h) \operatorname{sinc}([x-j h] / h)
$$

is exact if

$$
h \leqslant \pi / W \text {, }
$$

where $h$ is the grid spacing and $W$ is the bandwidth of $u_{W}(x)$ and where

$$
\operatorname{sinc}(x) \equiv \sin (\pi x) /(\pi x) \text {. }
$$

Lemma 3.10. The pseudospectral method using sinc basis functions and the grid points $x_{j}=j h$, $j=0, \pm 1, \pm 2, \ldots$, approximately solves $u_{x x}-u=f(\varepsilon x)$ by replacing the inhomogeneous function by $f_{W}(\varepsilon x)$ where $W=\pi / h$, and then solving this modified problem exactly.

What is remarkable about Theorem 3.7 is that for any error tolerance, no matter how small, we can always obtain that tolerance with a grid spacing which is large in comparison to 1 provided that $\varepsilon$ is sufficiently small. If $h \gg 1$, however, the pseudospectral method cannot possibly resolve variations of $u(x ; \varepsilon)$ on the fast, $\mathrm{O}(1)$ length scale. One can obtain very high accuracy anyway because the unresolved, fast scales have only an exponentially small amplitude.

When $\varepsilon$ is $O(1)$, then the variability on the $O(1)$ scale is not small and must be resolved. However, in this case, the "fast" and "slow" scales are indistinguishable. There is no numerical challenge and the pseudospectral algorithm will compute a very accurate approximation with a relatively small number of degrees of freedom.

In contrast, when $\varepsilon \ll 1$, the "fast" and "slow" scales are widely separated. Simultaneously resolving both length scales would require a huge number of grid points as noted earlier. The point of Theorem 3.7 is that when $\varepsilon \ll 1$, the fast scale does not have to be resolved.

The result is that regardless of whether $\varepsilon$ is small or not small, a moderate number of grid points are sufficient.

Of course, strictly speaking we have shown this only for Case 1 and only for the sinc pseudospectral method. Sections 6 and 7, however, present numerical evidence that confirms that exponentially small, fast scale variability is irrelevant to the rational Chebyshev pseudospectral method, too. For Case 2, Table 6 shows that the error in the radiation coefficient for a given number of grid points is almost independent of $\varepsilon$. For a hyperbolic secant forcing, $N=50$ points is sufficient for eight decimal place accuracy regardless of whether $\varepsilon$ is large or small.

\section{The multiple scales perturbation theory}

Theorem 4.1. The ordinary differential equation (1.1), $u_{x x} \pm u=f(\varepsilon x)$ on the interval $x \in[-\infty, \infty]$, has the asymptotic series solution

$$
u(X ; \varepsilon) \sim \pm \sum_{n=0}^{\infty}(\mp 1)^{n} \varepsilon^{2 n}\left(\mathrm{~d}^{2 n} / \mathrm{d} X^{2 n}\right) f(X),
$$


where $X$ denotes the "slow" coordinate

$$
X \equiv \varepsilon x .
$$

Proof. If we replace $x$ by $X$, assume that both $u$ and $f$ vary only on the slow length scale, and match powers of $\varepsilon$, we obtain (4.1). A full treatment of the method of "multiple scales" is given in the texts by Nayfeh [23] and Kevorkian and Cole [17].

A note on terminology: we shall refer to (4.1) as the "multiple scales" series even though the expansion actually depends only upon a single scale. We are forced to use this confusing label for two reasons. First, "multiple scales" is the chapter title and index heading where the method behind (4.1) is to be found in [17,23]. Second, there is no accepted terminology for that subset of multiple scales series where the usual "fast" scale variation is missing.

Multiple scales expansions, whether with "slow" and "fast" scales or just a "slow" scale as here, are usually divergent. We offer several pieces of evidence to show that (4.1) is normally divergent, too. Table 3 displays the first few terms of the series for the particular forcing, $f(\varepsilon x)=\operatorname{sech}(\varepsilon x)$. The rapid growth of the numerical coefficients (roughly as $(2 n) !)$ is a symptom of the series' divergence.

Theorem 4.2. The multiple scales perturbation series (4.1) is term-by-term identical with the series obtained by expanding the $1 /\left(1 \mp k^{2}\right)$ factor in the Fourier integral representation of the solution, (2.1), as a geometric series, that is,

$$
u(x ; \varepsilon) \sim \pm 1 \sum_{n=0}^{\infty}( \pm 1)^{n} \varepsilon^{2 n} \int_{-\infty}^{\infty} k^{2 n} F(k / \varepsilon) \mathrm{e}^{\mathrm{i} k x} \mathrm{~d} k / \varepsilon(2 \pi)^{1 / 2}
$$

Proof. Application of the geometric series to (2.1) coupled with the identity that the transform of the $2 n$th derivative of $f(\varepsilon x)$ is proportional to $k^{2 n} F(k / \varepsilon)$.

The theorem strengthens the case for the divergence of the perturbation series: the geometric expansion for $1 /\left(1 \mp k^{2}\right)$ converges only for $|k|<1$, but the integrals in (4.3) extend all the way to infinity. If one applies a power series beyond its radius of convergence, one must expect bad things to happen (like divergence).

Another way of understanding the series divergence is to note that the assumption that $u(x ; \varepsilon)$ varies only on the "slow" length scale is equivalent to the assumption that the Fourier

Table 3

The first terms in the perturbative solution of the wave equation for the special case $f(\varepsilon x)=\operatorname{sech}(\varepsilon x)$; here the variable $z \equiv \tanh (\varepsilon x)$ and $u^{(n)}$ is the coefficient of $\varepsilon^{n}$

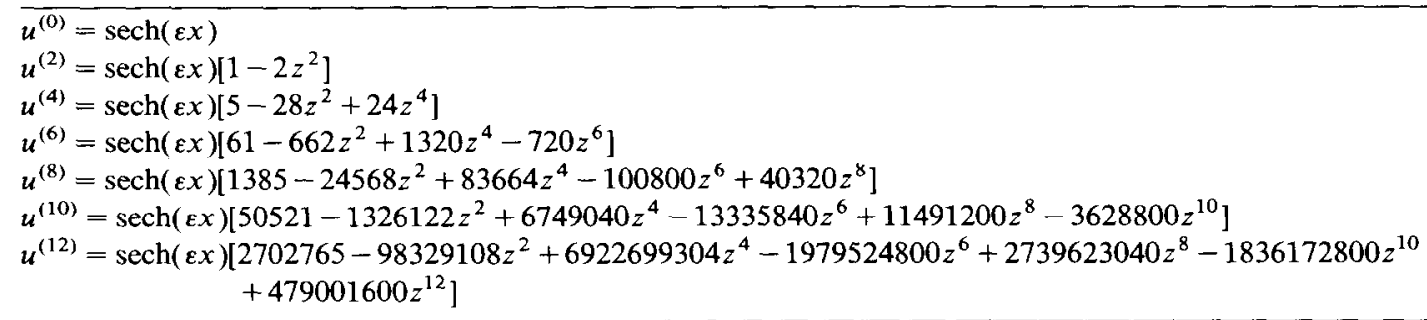


transform of $u(x ; \varepsilon)$ is restricted to wavenumbers such that $|k| \sim \mathrm{O}(\varepsilon)$. Were this true, then the power series expansion of the factor of $1 /\left(1 \mp k^{2}\right)$, which gives (4.3), would be convergent over the whole range of the integrand.

Unfortunately, the Fourier transform of most functions is nonzero for almost all wavenumbers. For example, if $f(\varepsilon x)=\operatorname{sech}(\varepsilon x)$, then the integrand of the Fourier transform representation of $u(x ; \varepsilon)$ is proportional to $\operatorname{sech}(\pi k / 2 \varepsilon)$. This vanishes only at infinity. Series (4.1) and (4.3) diverge because the Fourier transform is nonzero even for large $k$.

However, the contributions for $|k| \geqslant 1$ are the "fast scale variability" which Theorem 3.6 shows is exponentially small. For example, $\operatorname{sech}(\pi k / 2 \varepsilon)$ decays exponentially with $k$. Because the "tail" of this function and other Fourier transforms is exponentially small, (4.1) (and (4.3)) are asymptotic. When this series is truncated at the optimum order, the error in the asymptotic series is only $\mathrm{O}(\exp [-\mu / \varepsilon])$, the magnitude of the unresolved fast scale variability.

One might wonder if we could eliminate the divergence by employing a multiple scales perturbation theory with two scales. Unfortunately, this is possible only when the fast scale variability is $\mathrm{O}(1)$, not an exponentially tiny correction. The only perturbative procedure that has been successful is to apply a different technique, the method of matched asymptotic expansions in the complex $x$-plane, as done by Segur and Kruskal [26] and Pomeau, Ramani, and Grammaticos [25].

We shall not include complex-plane matched asymptotics in our discussion here because these perturbative solutions (for nonlinear waves) are compared with the Chebyshev method of [10, Section 7] and [12, Section 7], respectively. The conclusion is that the matched asymptotics series is accurate only for such small $\varepsilon$ that it is useful chiefly to prove the non-existence of classical solitons for these wave problems. The one advantage of the matched asymptotics series versus (4.1) is that the matched asymptotics analysis gives an approximation to the radiation coefficient $\alpha(\varepsilon)$. Except for $|x| \gg 1$, however, the matched asymptotics approximation is identical with (4.1). It therefore is important to analyze the errors in (4.1) more carefully.

Table 4 shows the errors in the multiple scales perturbation theory as a function of $\varepsilon$ and $n$, where $n$ is the number of terms retained in the series. For fixed $\varepsilon$, the error decreases to a minimum and then rises as $n$ increases, just as expected for any asymptotic series. (In Table 4, this pattern is clearest for $\varepsilon=0.2$.) Figure 2 shows something much more interesting: the

Table 4

Errors in perturbation theory as a function of $\varepsilon$ and $n^{\text {a }}$

\begin{tabular}{llll}
\hline$n$ & $\varepsilon=0.1$ & $\varepsilon=0.2$ & $\varepsilon=0.5$ \\
\hline 0 & 0.011 & 0.052 & 0.27 \\
1 & 0.00058 & 0.012 & 0.27 \\
2 & 0.000084 & 0.0063 & 0.32 \\
3 & 0.000023 & 0.0061 & 0.74 \\
4 & 0.0000096 & 0.0061 & 3.71 \\
5 & 0.0000053 & 0.0078 & 34.8 \\
6 & 0.0000047 & 0.015 & 471.0 \\
$\alpha$ & 0.0000047 & 0.0061 & 0.27 \\
\hline
\end{tabular}

"In each case, the maximum pointwise error or " $L_{\infty}$ norm" error is listed. Because the radiation coefficient $\alpha$ closely approximates the error of the optimum perturbation order, it is also given. The $n$ th-order term is proportional to $\varepsilon^{2 n}$. 


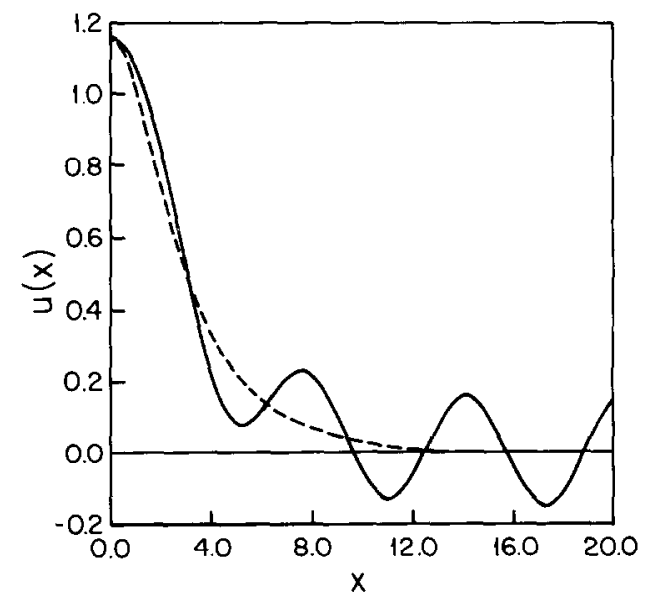

Fig. 2. Solid curve: $u(x ; \varepsilon=0.4)$ for $f(\varepsilon x) \equiv \operatorname{sech}(\varepsilon x)$. Dashed curve: optimum order perturbation series (zeroth-order plus $\left.O\left(\varepsilon^{2}\right)\right)$.

perturbation series gives a good approximation to the core of the solution (where $u(x ; \varepsilon) \approx f(\varepsilon x)$ ), but is a terrible approximation to the oscillatory "wings". The graph suggests what is confirmed by the table: the minimum error in the perturbation series is $\mathrm{O}(\alpha)$, where $\alpha$ is the amplitude of the oscillations in the "wings".

We can gain another perspective on the breakdown of the perturbation series by noting that $u^{(2 n)}(\varepsilon x)$, the coefficient of $\varepsilon^{2 n}$ in the asymptotic series (4.1), satisfies an equation of the same form as the original problem with a different forcing function, i.c.,

$$
u_{x x}^{(2 n)} \pm u^{(2 n)}=f^{(2 n)}
$$

where $f^{(2 n)}(\varepsilon x)$ is the second derivative of $u^{(2 n-2)}$. To solve (4.4), we drop the second derivative term (which is $\mathrm{O}\left(\varepsilon^{2}\right)$ smaller than the undifferentiated term) and therefore

$$
u^{(2 n)}= \pm f^{(2 n)} \text {. }
$$

Thus, the $n$ th-order forcing and the $n$ th-order term in the asymptotic series for $u(x ; \varepsilon)$ are identical except perhaps for a sign.

Figure 3 graphs the shape of $f^{(2 n)}(\varepsilon x)$ for $n=1,2, \ldots, 6$ for $f(\varepsilon x)=f^{(0)}=\operatorname{sech}(\varepsilon x)$. It follows from (4.5) that these terms are, to within a sign, merely the $u^{(2 n)}(x)$ whose analytical expressions are given in Table 3. The crucial point is that the multiple scales assumption-dropping the second derivative to pass from (4.4) to (4.5) - is justified only as long as both the forcing and the correction vary with $x$ only on the "slow", $\mathrm{O}(1 / \varepsilon)$ length scale. Figure 3 shows that $f^{(2 n)}(x)$ varies more and more rapidly as $n$ increases. For example, each $f^{(2 n)}(x)$ has a maximum at $x=0$ and roots nearest the origin at approximately $x \approx \pm 1 / n \varepsilon$. Thus, for $\varepsilon=0.2$, which is used to scale the $x$-axis in Fig. $3, f^{(12)}(x)$ has roots at approximately \pm 1 , which implies that this forcing is varying as rapidly as the homogeneous solutions of (1.1). The assumption of separation of scales has failed and we cannot legitimately approximate (4.4) by (4.5) for $n$ this large for this $\boldsymbol{\varepsilon}$.

Table 4 confirms this by showing that, for $\varepsilon=0.2$, the error in the asymptotic series is a minimum for $n=4$ and increases for larger $n$. Standard texts on perturbation theory such as [23] 


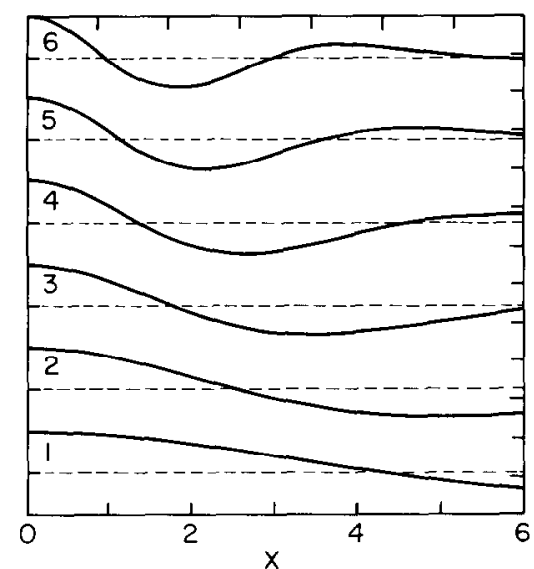

Fig. 3. A graph of the shapes of the forcing functions, $f^{(2 n)}(\varepsilon x)$, for $n=1$ (bottom) to $n=6$ (top) for the case where $f^{(0)}(\varepsilon x)=\operatorname{sech}(\varepsilon x)$. The theme of the graph is that the forcing varies more and more rapidly with $x$ as $n$ increases, invalidating the multiple scales perturbation theory for sufficiently large $n$ for a given $\varepsilon$. The functions have been scaled by dividing out the common factor of $\operatorname{sech}(\varepsilon x)$ and a constant so that their spatial variability is easier to see. The $x$-axis scale assumes $\varepsilon=0.2$, but the plots apply to all $\varepsilon$ with appropriate rescaling of this axis. The graph applies to both Cases 1 and 2 because the forcing functions are independent of the sign in (1.1) except for sign. These graphs, again except possibly for sign, also illustrate the perturbative terms, $u^{(2 n)}$, whose analytical terms are listed in Table 3 : $f^{(2 n)}= \pm u^{(2 n)}$ for all $n$ and both Case 1 and Case 2

invariably quote the rule of thumb that one should truncate an asymptotic series at that order where the correction is smallest.

Figure 3 suggests an alternative stopping criterion: graph the forcing $f^{(2 n)}(\varepsilon x)$ for various $n$ and stop at that order $n$ where $f^{(2 n)}(\varepsilon x)$ is no longer a slowly-varying function of $x$. This criterion is a little fuzzy in the sense that the judgment of what functions are "slowly-varying" and what functions are not is somewhat subjective. However, the alternative analytical criterion of truncating at the smallest term does not come with a guarantee either.

The next result gives a specific condition for divergence.

Theorem 4.3. If $f(x)$ is singular in the complex plane for $|\operatorname{Im}(x)|=\mu$, then the multiple scales perturbation series (4.1) is divergent for all finite $\varepsilon$. Furthermore, at least an infinite subsequence of terms in the series must satisfy the inequality

$$
\max _{\text {in } x}\left|\left(\mathrm{~d}^{2 n} / \mathrm{d} X^{2 n}\right) f(X)\right|>(2 n) !(1 / \mu)^{2 n} .
$$

In words, the nth term of the series is diverging as $(2 n) !$. The $(1 / \mu)$ factor can slow the rate of divergence, but never stop it.

Proof. The terms in the multiple scales series (4.1) are the coefficients of the even terms in the Taylor expansion (in $x$ ) of $f(\varepsilon x)$ about $x=0$ except that the Taylor coefficients are divided by $(2 n)$ !. If $f(x)$ has singularities at $|\operatorname{Im}(x)|=\mu$, it follows that the radius of convergence of the power series is no greater than $\mu$ for some real value of $x$. This implies that at least a subsequence of terms must grow as fast as specified by (4.6). Otherwise, the Taylor series would have a radius of convergence greater than $\mu$, which is a contradiction. 
Theorem 4.4. Even if $f(x)$ is an entire function, that is, the width of its strip of analyticity is $\mu=\infty$, the multiple scales series may still diverge.

Proof. If $f(\varepsilon x)=\exp \left(-(1 / 2) \varepsilon^{2} x^{2}\right), \alpha$ is proportional to $\exp \left(-\left(1 /\left[2 \varepsilon^{2}\right]\right)\right.$. This cannot be represented by a convergent series in positive powers of $\varepsilon$, so clearly the perturbation series must diverge. By using the power series for the exponential, one can show that the terms in the perturbation diverge as $[(2 n) !]^{1 / 2}$, which is slower than as $(2 n) !$,but still implies a zero radius of convergence for (4.1).

Theorem 4.5. If $f(x)$ is a bandlimited function of bandwidth $W$, then the multiple scale series (4.1) converges for

$$
\varepsilon^{2}<1 / W
$$

for both Case 1 and Case 2.

Proof. If $f(x)$ has bandwidth $W$, then $F(k / \varepsilon)=0$ for $|k|>\varepsilon W$. Truncating the integrals in (4.2) to $k \in[-\varepsilon W, \varepsilon W]$ and replacing each factor in the integrand by its maximum on this interval implies that each integral in (4.2) is bounded by $\varepsilon^{4 n} W^{2 n} D$, where $D$ is a sufficiently large constant. Since this bounding series is a geometric series, it converges whenever (4.7) is true.

We thus gain a perspective on the multiple scales series which is not given in any textbook. For arbitrary $u(x)$, we can write

$$
u(x ; \varepsilon)=u_{w}(x ; \varepsilon)+\Delta(x),
$$

where the bandlimited function is defined by truncating the Fourier integral for $u(x ; \varepsilon),(2.1)$, at $k= \pm W$. When $\varepsilon$ is small, Theorem 4.5 shows that the multiple scales series for $u_{W}(x ; \varepsilon)$ is convergent. It follows that the divergence of the series for $u$ comes from entirely from the divergence of the series for $\Delta(x)$.

However, Lemma 3.8 (or equivalently, Theorem 3.6) shows that when $\varepsilon \ll 1, \Delta(x)$ is exponentially small in $1 / \varepsilon$. It follows that although the series for $\Delta(x)$ is divergent, its first few terms must be very, very tiny. Consequently, adding these nonsense terms to those of $u_{W}(x ; \varepsilon)$ will give a very good approximation to $u(x ; \varepsilon)$. If we add hundreds or thousands of terms of $\Delta(x)$, however, the factorial growth of these terms with $n$ will eventually swamp the "good" series for $u_{W}(x ; \varepsilon)$, and the sum of the two series, (4.1), will give rubbish.

The conclusion is that the multiple scales series is numerically useful for small $\varepsilon$. For moderate and large $\varepsilon$, however, the perturbation series is useless because it is divergent except under very unusual circumstances, such as when $f(\varepsilon x)$ is bandlimited. When $\varepsilon$ is not small, $\Delta(x)$ in $(4.8)$ is not small and we are stuck with a large error. In the next three sections, we discuss alternative numerical techniques that are not restricted to $\varepsilon \ll 1$.

\section{Padé approximants}

The $[M, N]$ Padé approximant to a function $f(\varepsilon)$ is a rational function whose numerator is a polynomial of degree $M$ in $\varepsilon$ and whose denominator is a polynomial of degree $N$. By 
convention, the two polynomials are divided by a common factor to normalize the constant in the denominator to 1 . The remaining $(M+N+1)$ degrees of freedom are determined by the requirement that the power series of the approximant should agree with that of $f(\varepsilon)$ through the first $(M+N+1)$ terms. The coefficients of the two polynomials may be calculated by solving a linear system of algebraic equations or by more efficient recursions.

One might suppose that such a rearrangement of one polynomial (the truncated power series) into the equivalent ratio of two polynomials would accomplish nothing. In reality, the Padé approximant is almost always more accurate than the truncated power series. Padé theory is thoroughly discussed in $[1 ; 2 ; 3$, p. 383].

Unfortunately, general Padé convergence theorems are restricted and limited. One exception is that when the Fourier transform of the forcing function is nonnegative, $u(0 ; \varepsilon)$ is a so-called "Stieltjes function" [3, p. 120]. (More precisely, the sum of two Stieltjes functions after the factor of $1 /\left(1 \mp k^{2}\right)$ is resolved into partial fractions.) Case 1, exponential decay, is the sum of two Stieltjes functions evaluated on the imaginary axis (after $\varepsilon$ is replaced by a new complex coordinate to convert these functions to standard form). One can rigorously prove that for all finite $\varepsilon$, the $[N, N]$ ("diagonal") Padé approximant converges from above (in magnitude) while the $[N-1, N]$ ("subdiagonal") converges monotonically from below.

Figure 4 verifies the proof. Since the diagonal Padé approximant is too large and the subdiagonal approximant is too small, the two have been averaged together. The reason that the logarithm of the error is plotted versus the square root of $N$ instead of $N$ itself is that for the Stieltjes function,

$$
S(\varepsilon) \equiv \int_{0}^{\infty} \mathrm{d} t \mathrm{e}^{-t} /(1+\varepsilon t) \sim \sum_{n=0}^{\infty}(-1)^{n} n ! \varepsilon^{n},
$$

it has been proved that error in both the diagonal and subdiagonal Padé sequences (and therefore in their average) is

$$
E(N ; \varepsilon) \sim q(\varepsilon, N) \mathrm{e}^{-4 N^{1 / 2} / \varepsilon^{1 / 2}}
$$

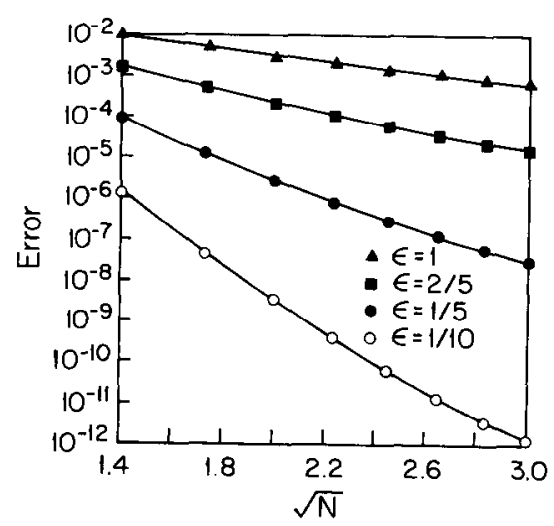

Fig. 4. A plot of the absolute error in the average of the $[N-1, N]$ and $[N, N]$ Padé approximants to $u(0 ; \varepsilon)$ for Case 1 (exponential decay; $u_{x x}-u=\operatorname{sech}(\varepsilon x)$ ). The four rightmost values for $\varepsilon=1 / 10$ have been slightly inflated by roundoff error; the average of the [8,9] and [9,9] approximants is probably more accurate than the $10^{-12}$ error shown. Since $u(0 ; \varepsilon) \approx 1$ for all values of $\varepsilon$ on the graph, the relative errors differ by no more than $30 \%$ from the absolute errors. 
where $q(\varepsilon, N)$ denotes a function that varies much more slowly with both $N$ and $\varepsilon$ than the exponential. The exponential in (5.2) would appear on the $\log$-square root plot as a straight line. Figure 4 shows that $u(0 ; \varepsilon)$ for Case 1 seems to asymptote to a straight line for all four values of $\varepsilon$ shown. This rate of convergence is "exponential but subgeometric with an index of convergence of $1 / 2$ " in the language of [6]. The pseudospectral methods discussed in the next section have similar rates of convergence.

Unfortunately, Case 2 is the sum of Stieltjes functions evaluated on their branch cuts. (For (5.1), the analogous task is to evaluate $S(\varepsilon)$ on the negative $\varepsilon$-axis where the integral representation has poles on the integration interval and where the terms in the perturbation series are all positive instead of alternating.) The Padé theory is inapplicable and Fig. 5 shows that the approximants fail, too. "Divergence" is not a good description of the way the Padé approximants fail: the error does not become unbounded in $N$, but oscillates. It is intriguing that the mean of the oscillations in $N$ is roughly equal to $\alpha(\varepsilon)$, which is shown on Fig. 5 as the horizontal dividing line for each $\varepsilon$.

This Padé failure is disappointing because small or zero $|x|$ is the only region where one could even hope that Padé approximants might succeed when $u(x ; \varepsilon)$ oscillates for large $|x|$. The reason is that the Padé approximants are constructed from perturbative terms which each decay exponentially as $|x| \rightarrow \infty$. For large $|x|$, the Padé approximant may asymptote to a constant or to zero, but it cannot mimic an infinite number of oscillations. Figure 6 shows what happens when we compare the Padé approximants with $u(x ; \varepsilon)$ for general $x$. Like the perturbation series, the Padé terms completely miss the far field waviness. In addition, all the approximants are bedeviled by poles for real $x$ that make their graphs jagged, ugly, and unusable.

Figure 7 shows that the corresponding Padé approximants for Case 1 are much better behaved. The average of $[2,3]$ and $[3,3]$, henceforth called the " $N=3$ averaged" approximation for short, faithfully tracks $u(x ; \varepsilon)$ for all $x$. The $N=5$ approximation is also good although the error is slightly larger than for $N=3$, perhaps because of roundoff error. (One of the unpleasant aspects of Padé methods is their vulnerability to roundoff. However, the recursive Padé

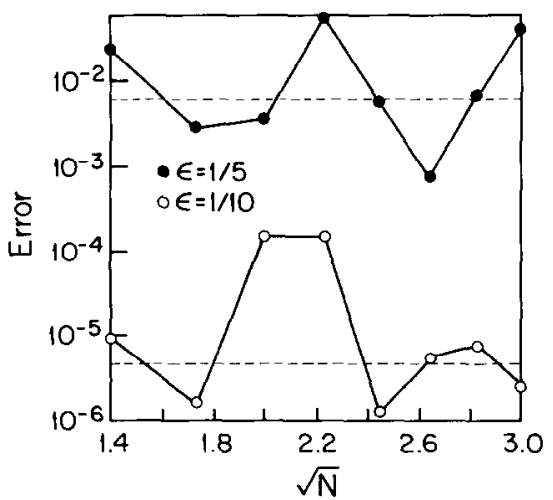

Fig. 5. The logarithm of the absolute error of the average of the $[N-1, N]$ and $[N, N]$ Padé approximants to $u(0 ; \varepsilon)$ for Case 2 (far field oscillations; $u_{x x}+u=\operatorname{sech}(\varepsilon x)$ ) for $\varepsilon=1 / 5$ (top graph) and $\varepsilon=1 / 10$, plotted versus $N^{1 / 2}$. The dashed horizontal dividing lines are the absolute values of $\alpha(1 / 5)$ and $\alpha(1 / 10)$, respectively. The approximants do not converge, but instead the error oscillates about the amplitude of the far field radiation, $\alpha(\varepsilon)$. 

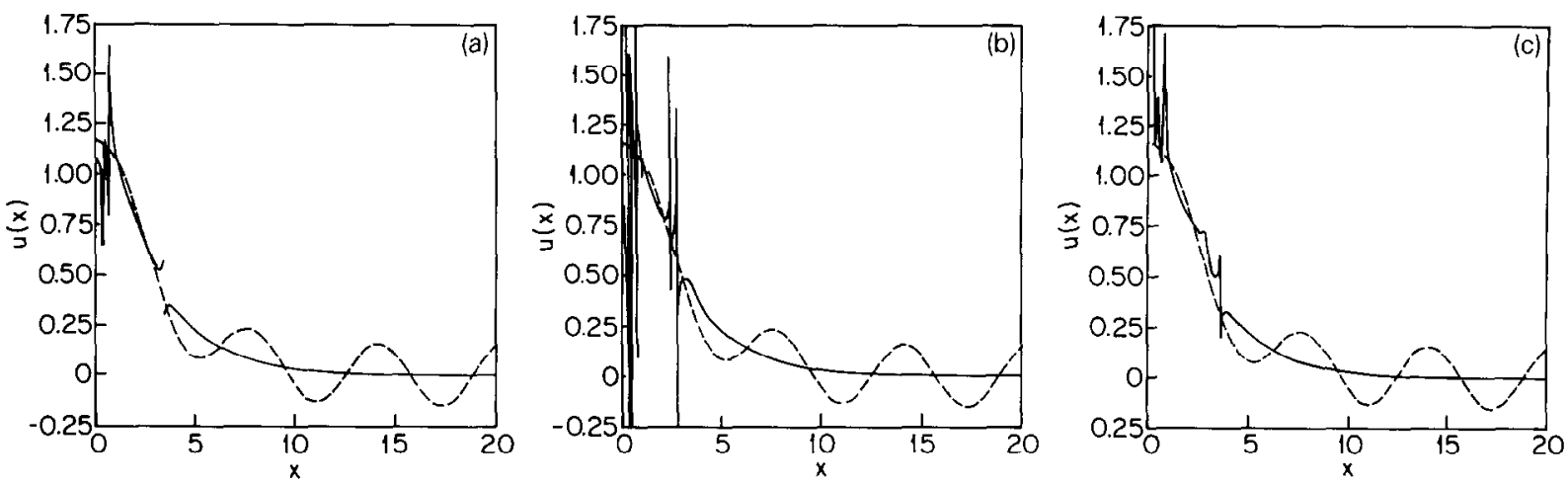

Fig. 6. The averages of the $[N-1, N]$ and $[N, N]$ Padé approximants (solid) are compared with the exact $u(x ; \varepsilon)$ (dashed) for $\varepsilon=0.4$ for Case 2 (far field oscillations). (a) $N=3$; (b) $N=4$ average; (c) $N=5$ average.

algorithms described in $[1,2]$ are more stable than the matrix-solving method used to make Fig. 7.)

The $N=4$ averaged approximation, alas, has a pair of poles near $x=5$. (Note that the Stieltjes theory applies only at $x=0$.) Unfortunately, this alarming behavior is typical even for successful applications of Padé approximants. For example, Baker [2, p. 122] shows that for the function

$$
t(\varepsilon) \equiv\left\{\left[\left(1+\varepsilon+\varepsilon^{2}\right)(1+2 \varepsilon)\right]^{1 / 3}-1\right\} / \varepsilon,
$$

the $[1,1],[4,4],[7,7], \ldots,[3 M+1,3 M+1]$ approximants do not even exist because the linear equations that match the approximants to the power series of $t(\varepsilon)$ are inconsistent. However, all the other diagonal Padé approximants exist and converge very rapidly for all complex $\varepsilon$ except at the branch points.

Similarly, [3, p. 400] shows that a Padé approximant may fail to converge even at a point

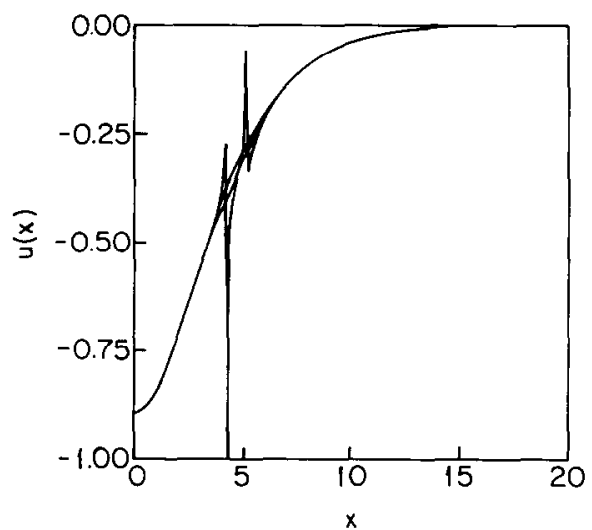

Fig. 7. Same as Fig. 6 but for Case 1: $u(x ; \varepsilon)$ decays exponentially for large $|x|$. The $N=3,4$, and 5 averaged Padé approximants all closely resemble the exact solution except for the spike in the $N=4$ approximant. Because these curves are so similar, the three approximants have been graphed together. 
where the function has no singularities. Nonetheless, with a little care, i.e., comparing many different $N$, Padé methods are very powerful.

The numerical evidence suggests that Padé approximants-or at least some subsequencesconverge for Case 1 (exponentially decaying solutions). For Case 2, however, the approximations neither converge nor diverge, but instead stall out, like the asymptotic series from which they are formed, at a minimum error which is roughly equal to the radiation coefficient $\alpha(\varepsilon)$.

\section{Numerical method I: orthogonal rational functions}

Boyd [4,5] and Grosch and Orszag [14] have shown that it is easy to solve boundary value problems on an infinite interval by using the so-called "rational Chebyshev functions", which are the images of the ordinary Chebyshev polynomials under a mapping. These same basis functions may be more compactly defined via

$$
\mathrm{TB}_{n}(x) \equiv \cos (n t), \quad n=0,1,2, \ldots
$$

under the mapping

$$
t=\operatorname{arccot}(x / L),
$$

where $L$ is a user-choosable constant map parameter. ( $L=2 / \varepsilon$ in the calculations described below.) Solving boundary value problems using these basis functions is thoroughly described with examples in [4].

There is one major problem: as discussed in [4,5], the rational Chebyshev series is useless when $u(x)$ is oscillatory for large $x$ unless the magnitude of $u(x)$ is very small. In practical terms, this means that the pseudospectral method with a basis composed purely of $\operatorname{TB}_{n}(x)$ is subject to the same limitations as the perturbation theory and Padé approximants: it is accurate only to the extent that the radiation may be ignored. In the next section, we describe a modification of the basis that overcomes this problem.

Here, we wish to show that the rational basis is successful for small $\varepsilon$ even for Case 2 . There is one technical complication. When the desired solution $u(x)$ decays exponentially as $|x| \rightarrow \infty-$ the usual case in applications of the $\mathrm{TB}_{n}(x)$ - the other linearly independent solution of the (second-order) differential equation normally increases exponentially with $x$ in the same limit. Thus, the decaying solution is the only bounded solution. In this case, the boundary condition of decay is "natural", that is to say, it is not necessary to impose any conditions on the basis functions. The $\mathrm{TB}_{n}(x)$ series will automatically converge to the correct, bounded solution of the differential equation for Case 1 (exponential decay).

For Case 2, however, the asymptotic behavior of the solutions of the differential equation is oscillatory rather than exponential. We now must force the numerical solution to be zero at infinity by replacing the rational Chebyshev functions by the new basis functions

$$
\begin{aligned}
& \phi_{2 n}(x) \equiv \mathrm{TB}_{2 n}(x)-1, \\
& \phi_{2 n+1}(x) \equiv \mathrm{TB}_{2 n+1}(x)-\mathrm{TB}_{1}(x)
\end{aligned}
$$

for $n \geqslant 1$. Since each basis function individually vanishes as $|x| \rightarrow \infty$, so, too, must the numerical solution which is their weighted sum. When $f(x)$ is symmetric, as is true of $\operatorname{sech}(\varepsilon x)$, then we may restrict the hasis to the even degree functions (i.e., (6.3a)). 
Table 5

Errors in numerical solution of $u_{x x}-u=\operatorname{sech}(\varepsilon x)$ for various $\varepsilon$ and $N^{\text {a }}$

\begin{tabular}{lll}
\hline$N$ & \multicolumn{2}{l}{$L_{\infty}$ errors } \\
\cline { 2 - 2 }$\varepsilon=0.1$ & $\varepsilon=0.5$ \\
\hline 6 & 0.0023 & 0.0041 \\
10 & 0.00013 & 0.00018 \\
15 & 0.000029 & 0.000042 \\
20 & 0.0000039 & 0.0000054 \\
30 & 0.00000017 & 0.00000022 \\
40 & 0.000000011 & 0.000000016 \\
50 & $0.27 \mathrm{E}-8$ & $0.38 \mathrm{E}-8$ \\
\hline
\end{tabular}

${ }^{a} N$ is the total number of symmetric rational Chebyshev basis functions; the highest degree included in the basis is $\phi_{2 N}(x)$. The $L_{\infty}$ error is $\max \left|u(x ; \varepsilon)-u_{N}(x ; \varepsilon)\right|$ for all $x$. The maximum absolute values of $u$ are $\max \mid u(x ; \varepsilon=$ $1 / 10) \mid=0.99$ and $\max |u(x ; \varepsilon=1 / 2)|=0.89$.

The pseudospectral interpolation points are

$$
x_{i} \equiv L \cot (\pi[2 i+1] /[2 N+2]), \quad i=1, \ldots, N,
$$

where $N$ is the number of basis functions which are retained in the truncation. As in all collocation methods, the differential equation is discretized by substituting the truncated spectral series into the equation and then demanding that the residual should be zero at each of the $N$ interpolation points, (6.4). This gives a dense system of $N$ linear equations in $N$ unknowns, which were solved by Gaussian elimination.

Table 5 demonstrates that this method is very successful for Case 1: exponential decay. One might suppose that the error would be large in the limit $\varepsilon \rightarrow \infty$ for fixed $N$ because the ratio of the "fast" and "slow" length scales becomes arbitrarily large. We have already shown in Section

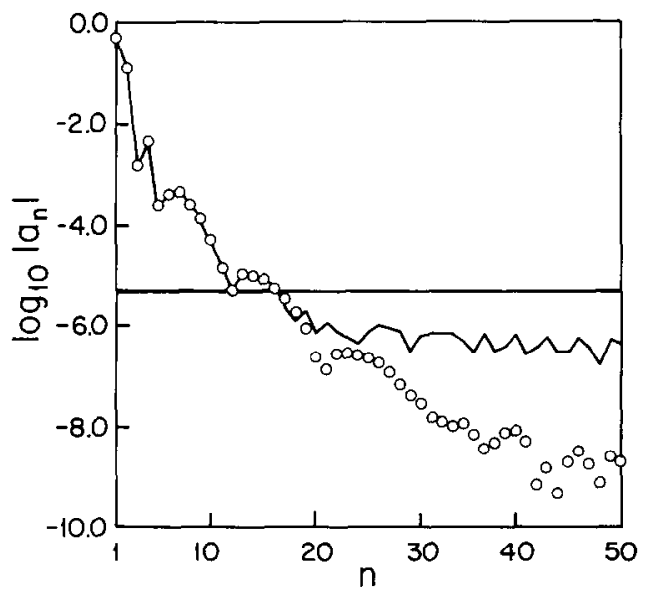

Fig. 8. The base-10 logarithm of the absolute values of the rational Chebyshev coefficients are plotted for two different numerical solutions for $\varepsilon=0.1, f(\varepsilon x)=\operatorname{sech}(\varepsilon x)$, and 50 pseudospectral degrees-of-freedom. The horizontal line indicates the logarithm of the radiation coefficient $\alpha$. Solid curve: pure $\operatorname{TB}_{n}(x)$ basis set. Symbols: TB $100(x)$ was replaced by the special "radiation hasis function". 
3 , however, that the magnitude of the rapidly-varying part of $u(x ; \varepsilon)$ is exponentially small in $1 / \varepsilon$ as $\varepsilon \rightarrow 0$. Consequently, it is not necessary to resolve the "fast" length scale-only the slow one-to obtain many decimal places of accuracy for $\varepsilon \ll 1$. Table 5 eloquently confirms this theoretical prediction.

For Case 2, Fig. 8 shows that for small $\varepsilon$, this naive method of ignoring the far field oscillation is quite effective. The coefficients differ from those of the more sophisticated method described below by less than the radiation coefficient $\alpha$, which is $4.7 \times 10^{-6}$ for $\varepsilon=0.1$. However, the $\left\{a_{n}\right\}$ for large $n$ level off at roughly one order-of-magnitude smaller than $\alpha$. It is clear that increasing the size of the basis set would produce no further improvement.

\section{Numerical method II: rational Chebyshev functions with radiation basis functions}

For Case 2, the perturbation theory and the pure Chebyshev basis both misrepresent the behavior of $u(x ; \varepsilon)$ at infinity by ignoring the sinusoidal radiation. Imposing the correct large $|x|$ behavior is subtle because, without making $u(x ; \varepsilon)$ unbounded, we can add

$$
\gamma \cos (x)+\delta \sin (x)
$$

to $u(x ; \varepsilon)$ for arbitrary constants $\gamma$ and $\delta$. For (1.1) and also for the variable coefficient equations discussed in Section 8, these two eigenfunctions are the homogeneous solutions of the differential equation.

This indeterminancy can only be resolved by the needs of the physical analysis. Lorenz and Krishnamurthy [18] choose

$$
\gamma=0 \quad \text { and } \quad \delta=\alpha \text {, }
$$

so that $u(x ; \varepsilon) \rightarrow 0$ as $x \rightarrow-\infty$ at the expense of doubling the far field oscillation as $x \rightarrow \infty$. In contrast, Hunter and Scheurle [16] choose

$$
\gamma=\delta=0
$$

which yields a capillary-gravity wave which is symmetric about the origin.

We shall use the same choice here. Equation (7.3) requires that the correct asymptotic behavior match that of the particular solution (2.3):

$$
u(x ; \varepsilon) \sim \alpha(\varepsilon) \operatorname{sgn}(x) \sin (x)+\beta(\varepsilon) \operatorname{sgn}(x) \cos (x) \text { as }|x| \rightarrow \infty
$$

for some constants $\alpha(\varepsilon)$ and $\beta(\varepsilon)$. (Note that because of the $\operatorname{sgn}(x)$ factors, neither term in (7.4) is a homogeneous solution of (2.1).)

To compute a numerical solution that satisfies (7.4), we modify the pure Chebyshev set by replacing one symmetric Chebyshev basis function by

$$
\phi_{\mathrm{s}, \mathrm{rad}} \equiv \tanh (\varepsilon x) \sin (x) \quad \text { ("symmetric radiation basis function") }
$$

and the highest antisymmetric basis function by

$$
\phi_{\mathrm{a}, \mathrm{rad}} \equiv \tanh (\varepsilon x) \cos (x) \quad \text { ("antisymmetric radiation function"). }
$$

The numerical approximation becomes

$$
u(x ; \varepsilon)=\sum_{n=2}^{N-1} a_{n} \phi_{n}(x)+\alpha \phi_{\mathrm{s}, \mathrm{rad}}(x ; \varepsilon)+\beta \phi_{\mathrm{a}, \mathrm{rad}}(x ; \varepsilon) .
$$


Table 6

Errors in the approximation of the "radiation" coefficient $\alpha$ by the coefficient of $\phi_{\mathrm{s}, \mathrm{rad}}(x)$ in the mixed Chebyshev/"radiation function" solution of the boundary value problem (1.1) with $N=50$

\begin{tabular}{llr}
\hline$\varepsilon$ & $\alpha_{\text {exact }}$ & Absolute error \\
\hline 0.1 & $4.73 \mathrm{E}-6$ & $2.00 \mathrm{E}-8$ \\
0.2 & $6.10 \mathrm{E}-3$ & $-5.94 \mathrm{E}-8$ \\
0.3 & 0.05573 & $1.82 \mathrm{E}-8$ \\
0.4 & 0.15469 & $2.44 \mathrm{E}-8$ \\
0.5 & 0.27102 & $2.41 \mathrm{E}-9$ \\
\hline
\end{tabular}

Table 7

Errors in the "radiation coefficient" for $\varepsilon=1 ; N$ is the number of (positive) collocation points; $(N-1)$ symmetric functions of the form $\left(\mathrm{TB}_{2 n}[x]-1\right)$ are combined with the single "radiation" function $\phi_{\mathrm{s}, \mathrm{rad}}$ to create the basis set

\begin{tabular}{rll}
\hline$N$ & $\alpha_{\text {approximate }}$ & Absolute error in $\alpha$ \\
\hline 2 & 3.59 & -2.962 \\
3 & 0.631 & -0.0055 \\
4 & 0.6270 & -0.00098 \\
5 & 0.62647 & -0.00044 \\
10 & 0.62591 & 0.00011 \\
15 & 0.62595 & 0.000075 \\
20 & 0.62601 & 0.0000078 \\
50 & 0.62602015 & -0.0000000068 \\
\hline
\end{tabular}

The rest of the procedure is the same as for any other pseudospectral method. The interpolation points are again given by (6.4); the sole change to accomodate the far field oscillations is the modification to the basis set.

The form of the radiation basis functions was dictated by several considerations. First, they must have the correct asymptotic behavior. Second, it is convenient that they have definite parity. $\operatorname{Tanh}(x)$ and $\sin (x)$ are both antisymmetric about $x=0$, so their product is symmetric, i.e., $\phi_{\text {s, rad }}(x)=\phi_{\text {s.rad }}(-x)$. Similarly, the other radiation function is antisymmetric with respect to the origin. If $f(\varepsilon x)$ has definite parity - $\operatorname{sech}(\varepsilon x)$, for example, is symmetric - then one may halve the basis set by including only those Chebyshev or radiation functions that have the same symmetry as the forcing function. Third, the width of the tanh function is chosen to be $O(1 / \varepsilon)$ so that the radiation basis function does not introduce any new length scales into the solution. One may, however, replace the hyperbolic tangent function by any smooth function that is antisymmetric, asymptotes to \pm 1 as $|x| \rightarrow \infty$, and has a width of $\mathrm{O}(1 / \varepsilon)$.

The mixed basis algorithm is extraordinarily accurate. For $f(\varepsilon x)=\operatorname{sech}(\varepsilon x)$, the computed coefficient of $\phi_{\mathrm{s} \text {, rad }}, \alpha$, is an $\mathrm{O}\left(10^{-8}\right)$ approximation to $\alpha$ for all $\varepsilon$. Figure 8 shows that instead of leveling off at a value set by $\alpha$, the Chebyshev coefficients in the mixed expansion (circles) continue to decrease with $n$. In startling contrast to both the perturbation series and the pure Chebyshev pseudospectral solution, the Chebyshev/radiation basis gives an error which is highly uniform in $\varepsilon$ as shown in Table 6. Table 7 shows that for a given fixed $\varepsilon$, the error decreases exponentially fast with $N$, as expected of a spectral algorithm.

This mixed basis pseudospectral method is equally effective for nonlinear problems. Numerical calculations for non-local waves include: the $\phi^{4}$ breather (scalar Higgs boson) [7,10,11], capillary-gravity water waves $[7,11,12]$ and ocean Rossby waves $[8,11]$.

\section{Variable coefficients}

To see what happens when the wave equation has variable coefficients, let us generalize (3.2) to

$$
u_{x x}+[1+p(\varepsilon x)] u=f(\varepsilon x) \text {. }
$$


Then $A(k)$, the Fourier transform of $u(x)$, is the solution of

$$
\left(1-k^{2}\right) A+(2 \pi)^{-1 / 2}(1 / \varepsilon) \int_{\infty}^{\infty} P(m / \varepsilon) A(k-m) \mathrm{d} m=(1 / \varepsilon) F(k / \varepsilon) .
$$

In the limit of small $\varepsilon$, the function $P(m / \varepsilon) / \varepsilon$ becomes increasingly tall and narrow like a delta-function. It is at least plausible that in this limit, there is only an exponentially weak coupling between the "slow" scale of $f(\varepsilon x)$ and $p(\varepsilon x)$ and the "fast" scale of the asymptotic radiation. However, a rigorous proof of this conjecture is beyond the scope of this work (and the author's mathematical skills!)

Instead, we numerically solved (8.2) to offer a non-rigorous proof-by-example. It is important to note that the nonlinear problems solved in [7,8,10-12] also have variable coefficients. The behavior shown in Fig. 9 for $p(\varepsilon x)=\operatorname{sech}(\varepsilon x)$ is generic.

As for the constant coefficient solutions, the radiation coefficient, $\alpha(\varepsilon)$, appears to asymptote to a straight line for large $1 / \varepsilon$. For this example, the sign of $\alpha(\varepsilon)$ changes twice over the range shown on the graph, which complicates the graph. Nevertheless, the trend of exponential decay with $1 / \varepsilon$ is certainly clear. It proved impractical to extend the graph to smaller $\varepsilon$ because $\alpha(\varepsilon=1 / 10) \leqslant 10^{-10}$. Variable coefficients do not restrict the applicability of the concepts and numerical methods developed here as long as the coefficients vary on the same slow scale as the forcing.

\section{Sensitivity to small perturbations}

The radiation coefficients $\alpha$ and $\beta$ are extremely sensitive to small perturbations. Table 2 shows that the radiation coefficient forced by $\varepsilon^{2 n} \operatorname{sech}^{2 n}(\varepsilon x)$ is independent of $n$ to lowest order in $\varepsilon$. However, the maximum value of $\varepsilon^{2 n} \operatorname{sech}^{2 n}(\varepsilon x)$ is only $\varepsilon^{2 n}$. This means that perturbations which are arbitrarily small can nonetheless control $\alpha$.

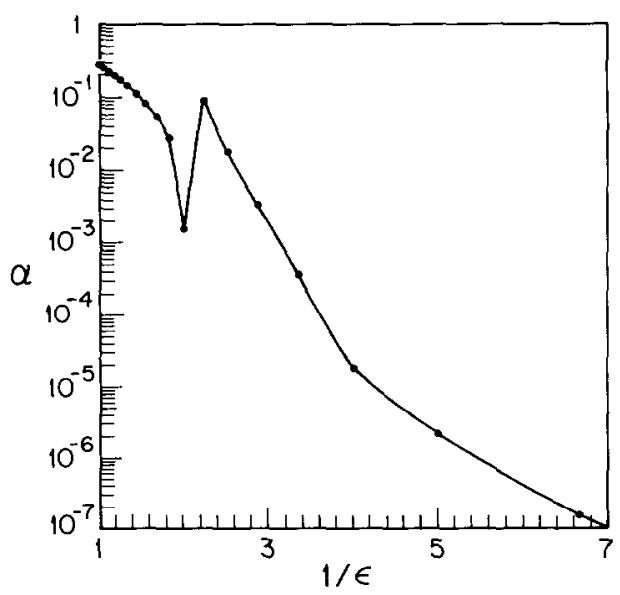

Fig. 9. A plot of the logarithm of the absolute value of the radiation coefficient $\alpha$ versus $1 / \varepsilon$ as obtained by the mixed $\mathrm{TB}_{n}(x) /$ radiation function numerical method for the differential equation $u_{x x}+(1+\operatorname{sech}[\varepsilon x]) u=\operatorname{sech}(\varepsilon x)$. If $\alpha \sim$ $O(\exp (-q / \varepsilon)$ as $1 / \varepsilon \rightarrow \infty$, as conjectured, then the curve should asymptote to a straight line. 
For example, if the forcing is

$$
f(\varepsilon x)=\operatorname{sech}^{2}(\varepsilon x)-362880 \varepsilon^{8} \operatorname{sech}^{10}(\varepsilon x)
$$

the radiation coefficient is

$$
\begin{aligned}
& \alpha-\left(\pi / \varepsilon^{2}\right) \mathrm{e}^{-\pi /(2 \varepsilon)}\left\{1-362880 / 362880+\mathrm{O}\left(\varepsilon^{2}\right)\right\} \quad(\varepsilon \ll 1) \\
& \quad \sim 0 .
\end{aligned}
$$

Thus, the two terms in (9.1) generate contributions to the radiation coefficient which almost cancel. (The cancellation is exact if the $\operatorname{sech}^{10}(\varepsilon x)$ factor is divided by $\left(1+4 \varepsilon^{2}\right)\left(1+16 \varepsilon^{2}\right)(1+$ $\left.36 \varepsilon^{2}\right)\left(1+64 \varepsilon^{2}\right) \sim 1$.) Nevertheless, the maximum of the second factor is smaller than the maximum of the first term by a factor of 300 when $\varepsilon=1 / 10$ and by a factor of 70,000 (!) when $\varepsilon=1 / 20$. One can make the second term arbitrarily small in comparison to the first by either taking smaller $\varepsilon$ or using a higher power of $\operatorname{sech}(\varepsilon x)$.

The analytical reason why the tiny second term can cancel the first is that $\operatorname{sech}^{10}(\varepsilon x)$ has a tenth-order pole at $x= \pm \pi /(2 \varepsilon)$ whereas $\operatorname{sech}^{2}(\varepsilon x)$ has only second-order poles at these same points. The heuristic reason is that $\operatorname{sech}^{10}(\varepsilon x)$ decays much more rapidly with $|x|$ than $\operatorname{sech}^{2}(\varepsilon x)$; the former is much more effective than the latter at contributing to the far field oscillations because its scale of variation is closer to the $O(1)$ length scale of the far field oscillations. One must be careful not to press this heuristic argument too closely, however. The theory of Fourier transforms shows that the order of the pole has only an algebraic effect on $\alpha$ (i.e., increasing the order of the pole by one increases $\alpha$ by a multiplier of $1 / \varepsilon$ ) while the location of the pole has an exponential effect on $\alpha$ as shown by Theorem 3.2.

This sensitivity to small perturbations is a very important limitation for solving weakly non-local nonlinear problems. For the capillary-gravity water waves of $[7,11,12]$ and the scalar Higgs boson of [7,10,11], the nonlinear multiple scales series has its $n$th term dominated by $\varepsilon^{2 n} \operatorname{sech}^{2 n}(\varepsilon x)$. One might imagine that it would be possible to calculate the radiation coefficient by modifying the strict multiple scales theory by solving (1.1) with a forcing function derived from the first few terms of the nonlinear series, i.e.,

$$
f(\varepsilon x)=\sum_{n=1}^{\infty} b_{2 n} \varepsilon^{2 n} \operatorname{sech}^{2 n}(\varepsilon x)
$$

Table 2 shows that the corresponding radiation coefficient is

$$
\alpha=\pi \mathrm{e}^{-\pi /(2 \varepsilon)}\left\{1+\mathrm{O}\left(\varepsilon^{2}\right)\right\} \sum_{n=1}^{\infty} b_{2 n} /(2 n-1) !
$$

Thus, all terms in the perturbation series for $f(\varepsilon x)$ contribute at the same order in $\varepsilon$. Worse still, the nonlinear multiple scales series, like that in Section 4, is asymptotic because the coefficients $b_{2 n}$ diverge as $\mathrm{O}(2 n !)$. The series in (9.4) need not even converge!

The conclusion is that for nonlinear problems, the analytical solution to (1.1) is not enough. Segur and Kruskal [26] and Pomeau, Ramani and Grammaticos [25] have shown that one may compute $\alpha$ by applying matched asymptotic expansions in the complex $x$-plane, but only in the limit $\varepsilon \rightarrow 0$. For computing $\alpha$ for nonzero $\varepsilon$, the Chebyshev/radiation function method is the only option. 


\section{Summary and conclusions}

In order to explain the concepts as simply and concisely as possible, we have ruthlessly restricted the problem to a linear ordinary differential equation in most of this article. However, the methods and analysis have a very wide range of applicability. In the previous section, we have already seen that the mixed Chebyshev/radiation algorithm (and the pure Chebyshev pseudospectral method, if $u(x ; \varepsilon)$ rapidly decays with $|x|)$ work just fine for equations with variable, rather than constant, coefficients. The only restriction is that the coefficients must vary on the same slow length scale as the forcing $f(\varepsilon x)$.

The second extension is to partial differential equations. There may be subtleties in two dimensions because the Laplacian operator and its solutions, for example, both asymptote to their limits algebraically fast (rather than exponentially fast) as the polar radius $r\left(=\left[x^{2}+\right.\right.$ $\left.\left.y^{2}\right]^{1 / 2}\right) \rightarrow \infty$. The $J_{n}$ Bessel functions, for instance, are asymptotically

$$
J_{n}(r)-[\text { constant }](1 / r)^{1 / 2} \cos (\phi[r]), \quad r \rightarrow \infty,
$$

where $\phi(r) \sim 1+\mathrm{O}\left(1 / r^{2}\right)$. The amplitude and phase factors in (10.1), because they are slowly varying instead of constant, are more challenging than the one-dimensional solutions described above where the difference between $u(x ; \varepsilon)$ and a simple trigonometric or exponential function is exponentially small in $|x|$. Nevertheless, Boyd [5] shows how one can compute very accurate spectral approximations to (10.1), so these difficulties are not serious. For the $\phi^{4}$ breather [10], however, the one-dimensional methods of this article extend to two dimensions without modification.

The third extension is to nonlinear equations. Boyd [8,10-12] applies the ideas and numerical algorithms developed here to nonlinear waves: the $\phi^{4}$ breather and capillary-gravity water waves. Although a full description would take us too far afield, two points are crucial.

The first is that the nonlinear problem is solved by a multiple scales expansion just like that for (10.1). Although one might suppose that the breather series diverges (as it is known to do [10]) because of the devil of nonlinearity, it is actually the method of multiple scales-and its neglect of linear derivative terms - that makes the $\varepsilon$-series divergent, exactly as for (1.1).

The second point is that the mixed Chebyshev/radiation pseudospectral method and other concepts apply with remarkably little modification because of the smallness of the far field radiation. If $\alpha(\varepsilon)$ is tiny - and because the radiation coefficient is an exponential function of $1 / \varepsilon, \alpha(\varepsilon)$ will be very small even if $\varepsilon$ is only $\mathrm{O}(1 / 5)$ - then the wave dynamics will be linear to a very high degree of approximation in the far field. For large $|x|$, one may make a perturbative analysis in powers of $\alpha(\varepsilon)$ to justify applying the linear ideas developed here. The $\alpha$-expansion also provides a systematic means of refining the radiation basis set so that one can calculate the nonlinear wave to arbitrary precision.

Thus, the concepts and algorithms of this article, despite the almost embarrassing simplicity of (1.1), have a very wide applicability. This linear ordinary differential equation illuminates the subtleties of the method of multiple scales and why it fails.

The problem (1.1) also emphasizes the crucial importance of the sign on the asymptotic behavior of the solution. The differential equation $u_{x x}-u=f(\varepsilon x)$ has a solution which decays rapidly for large $|x|$ (Case 1). Padé approximants formed from the perturbation series are convergent. For the differential equation $u_{x x}+u=f(\varepsilon x), u(x ; \varepsilon)$ is oscillatory as $|x| \rightarrow \infty$. Padé approximants fail for Case 2. Numerically, we need no special tricks for Case 1: the 
rational Chebyshev pseudospectral method of Boyd [4,5] is uniformly accurate as $\varepsilon \rightarrow 0$. This is surprising because $u(x ; \varepsilon)$ has multiple length scales, but we explained in Section 3 (and demonstrated in Section 6 and Table 5), this is irrelevant. For Case 2, however, we need the special trick of the radiation-modified Chebyshev basis which is described in Section 7.

These are all useful accomplishments, but much remains for the future. One open problem is to develop a rigorous theory (as opposed to empirical numerical experiments) for mixed series of Chebyshev and radiation basis functions.

\section{Appendix A. The large time solution of the forced wave equation}

The wave equation is

$$
U_{x x}-U_{t t}=f(x) \cos (t)
$$

We lose no generality by specifying a unit frequency because one may always rescale time via $T=\omega t$ to make $\omega=1$, and then rescale $x$ and $f(x)$ to eliminate the leftover factors of $\omega^{2}$ and convert the equation into (A.1). It is trivial to show that if $u(x)$ is assumed proportional to $\cos (t)$ for all $t$, then one may separate variables by writing

$$
U(x, t)=u(x) \cos (t)
$$

where $u(x)$ satisfies the boundary value problem (1.1). It turns out, however, that the wave equation (A.1) evolves to a separable solution of the form of (A.2) from arbitrary initial conditions.

One may show this by solving (A.1) via Laplace transform from an arbitrary initial condition such as

$$
U(x, 0)=0 \text { and } U_{t}(x, 0)=0
$$

with the boundary conditions

$$
U( \pm \infty, t)=0, \quad t<\infty .
$$

The wave "forgets" the initial conditions as $t \rightarrow \infty$ at a given $x$, so the precise choice of $U(x, 0)$ and $U_{t}(x, 0)$ is not important. For (A.1) and (A.3), the exact solution for all time is

$$
U(x, t)=\cos (t) \tilde{u}(x, t)+\sin (t) \tilde{v}(x, t),
$$

where

$$
\begin{aligned}
& \tilde{u}(x, t)=\frac{1}{2}\left\{\int_{0}^{t} f(w+x) \sin (w) \mathrm{d} w-\int_{-t}^{0} f(w+x) \sin (w) \mathrm{d} w\right\} \\
& \left.\tilde{v}(x, t)=-\frac{1}{2} \int_{-t}^{t} f \overline{(w}+x\right) \cos (w) \mathrm{d} w .
\end{aligned}
$$

Figure A.1 is a schematic snapshot of $U(x, t)$ for some large time. As waves radiate left and right away from the origin, there is an undisturbed region for large $|x|$ (marked "IV") in the figure and then a wavefront ("III") at $|x|=t$. Within the wavefront, however, $U(x, t)$ tends to a steady periodic oscillation. In this area ("I" and "II"),

$$
\begin{aligned}
& \tilde{u}(x, t) \rightarrow u(x) \quad \text { (solution of (1.1)), } \\
& \tilde{v}(x, t) \rightarrow v(x)=-\alpha \cos (x)-\beta \sin (x),
\end{aligned}
$$




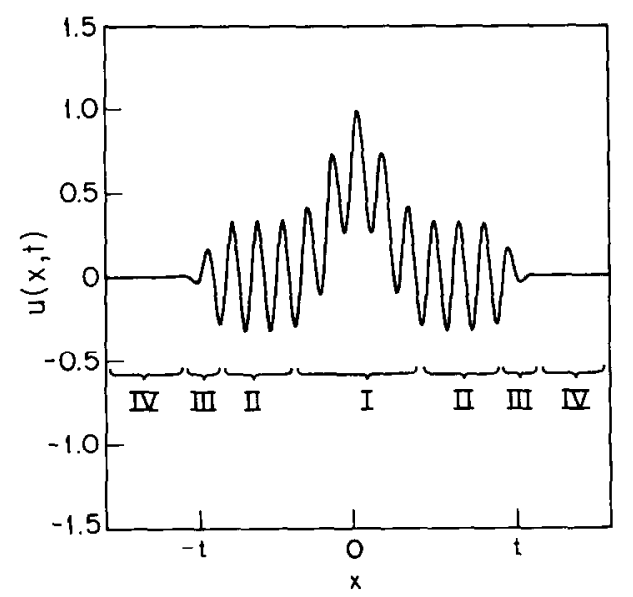

Fig. A.1. Schematic of the exact solution of (1.1) for large but finite time with the initial conditions $u(x, 0)=u_{t}(x, 0)$ $=0$. The four marked regions have the following names: (I) "directly-forced"; (II) "radiation"; (III) "wavefront"; (IV) "undisturbed". The "directly-forced" region is all $x$ such that $f(x)$ is nonnegligible. The "radiation" region is all $x$ such that $f(x) \approx 0$ and $|x|<\mathrm{t}$.

as $t \rightarrow \infty$, for fixed $x$, where $u(x)$ solves (1.1) and is given exactly by (2.3) and where the $\alpha$ and $\beta$ in $v(x)$ are the same radiation coefficients that appear in the asymptotics of (2.3).

Thus, although we have solved only a boundary value problem in this article, the solution is very relevant to the wave equation. Once $u(x)$ has been computed, it is trivial to add contribution of $\sin (t) v(x)$ to obtain the complete solution to the wave equation.

\section{Acknowledgement}

This work was supported by the National Science Foundation through grants OCE8509923, OCE8812300 and DMS8716766.

\section{References}

[1] G.A. Baker Jr, Advances in Theoretical Physics, Vol. 1 (Academic Press, New York, 1965).

[2] G.A. Baker Jr, Essentials of Padé Approximants (Academic Press, New York, 1975).

[3] C.M. Bender and S.A. Orszag, Advanced Mathematical Methods for Scientists and Engineers (McGraw-Hill, New York, 1978).

[4] J.P. Boyd, Spectral methods using rational functions on an infinite interval, J. Comput. Phys. 69 (1987) 112-142.

[5] J.P. Boyd, Orthogonal rational functions on a semi-infinite interval, J. Comput. Phys. 70 (1987) 63-88.

[6] J.P. Boyd, Chebyshev and Fourier Spectral Methods (Springer, New York, 1989).

[7] J.P. Boyd, New directions in solitons and nonlinear periodic waves: polycnoidal waves, imbricated solitons, weakly non-local solitary waves and numerical boundary value, algorithms, in: T.-Y. Wu and J.W. Hutchinson, eds., Advances in Applied Mechanics 27 (Academic Press, New York, 1989) 1-82.

[8] J.P. Boyd, Weakly non-local solitary waves, in: J.C.J. Nihoul and B.M. Jamart, eds., Mesoscale/Synoptic Coherent Structures in Geophysical Turbulence: Proceedings Twentieth Liege Colloquium on Hydrodynamics (North-Holland, Amsterdam, 1989) 103-112. 
[9] J.P. Boyd, A Chebyshev/radiation function pseudospectral method for wave scattering, Comput. Phys. 4 (1990) 83-85.

[10] J.P. Boyd, Numerical calculation of a weakly non-local solitary wave: the "breather" of the $\phi^{4}$ field theory, Nonlinearity 3 (1990) 177-195.

[11] J.P. Boyd, Weakly non-local solitary waves, in: A.R. Osborne and L. Bergamasco, eds., Proceedings Enrico Fermi School: Nonlinear Topics in Ocean Physics (North-Holland, Amsterdam, 1991).

[12] J.P. Boyd, Weakly non-local solitons for capillary-gravity waves: the fifth-degree Korteweg-de Vries equation, Phys. D 48 (1991) 129-146.

[13] R. Combescot, T. Dombre, V. Hakim, Y. Pomeau and A. Pumir, Shape selection of Saffman-Taylor fingers, Phys. Rev. Lett. 56 (1986) 2036-2039.

[14] C.E. Grosch and S.A. Orszag, Numerical solution of problems in unbounded regions: coordinate transforms, $J$. Comput. Phys. 25 (1977) 273-296.

[15] D.C. Hong and J.S. Langer, Analytic theory of the selection mechanism in the Saffman-Taylor problem, Phys. Rev. Lett. 56 (1986) 2032-2035.

[16] J.K. Hunter and J. Scheurle, Existence of perturbed solitary wave solutions to a model equation for water waves, Phys. D 21 (1988) 253-268.

[17] J. Kcvorkian and J.D. Colc, Perturbation Methods in Applied Mathematics (Springer, New York, 1981).

[18] E.N. Lorenz and V. Krishnamurthy, On the nonexistence of a slow manifold, J. Atmos. Sci. 44 (1987) 2940.

[19] C. Lozano and R.E. Meyer, Leakage and response of waves trapped by large islands, Phys. Fluids 19 (1976) 1075-1088.

[20] J.D. Meiss and W. Horton, Solitary drift waves in the presence of magnetic shear, Phys. Fluids 26 (1983) 990-997.

[21] R.E. Meyer, Exponential asymptotics, SIAM Rev. 22 (1980) 213-224.

[22] P.M. Morse and H. Feshbach, Methods of Theoretical Physics, Part I (McGraw-Hill, New York, 1953$) 495$.

[23] A. Nayfeh, Perturbation Methods (Academic Press, New York, 1973).

[24] V.L. Pokrovskii and I.M. Khalatnikov, On the problem of above-barrier reflection of high-energy particles, Soviet Phys. JETP 13 (1961) 1207-1210.

[25] Y. Pomeau, A. Ramani and B. Grammaticos, Structural stability of the Korteweg-de Vries solitons under a singular perturbation, Phys. D 21 (1988) 127-134.

[26] H. Segur and M. Kruskal, Nonexistence of small amplitude breather solutions in $\phi^{4}$ theory, Phys. Rev. Lett. 58 (1987) $747-750$.

[27] F. Stenger, Numerical methods based on Whittaker cardinal or sinc functions, SIAM Rev. 23 (1982) $165-224$. 